\title{
Disseminated Herpes Zoster Virus in a Patient With COVID-19
}

Kinjal Solanki, MD' - Kevin Kohm, MD² - Pinki J. Bhatt, MD'

A 64-year-old healthy man with a history of childhood chickenpox was brought in by ambulance for dyspnea and hypoxemia requiring supplemental oxygen via nasal cannula.

\section{History}

He reported generalized fatigue for 2 weeks prior to admission to the emergency department with worsening dyspnea for 1 day. He did not have any associated fevers, chills, chest pain, cough, hemoptysis, hematemesis, or melena. He denied any recent travel.

\section{Physical examination}

He had tachypnea, but the rest of the physical examination was unremarkable. His vital signs revealed a normal temperature of $36.2^{\circ} \mathrm{C}$, an elevated heart rate of $105 \mathrm{~b} / \mathrm{min}$, a low blood pressure of $100 / 54 \mathrm{~mm} \mathrm{Hg}$, an elevated respiratory rate of 40 breaths/min, and an oxygen saturation level of $80 \%$ on $6 \mathrm{lpm}$ nasal cannula, for which he was eventually intubated.

\section{Laboratory testing}

His white blood cell count and hemoglobin level were normal. However, the patient's laboratory test results were significant for a low platelet count of 128,000 per $\mathrm{mcL}$ (reference range, 140,000-440,000 per $\mathrm{mcL}$ ), a low absolute lymphocyte count of $750 / \mathrm{uL}$ (reference range, 850-3000/uL), an elevated D-dimer level of $2014 \mathrm{ng} / \mathrm{mL}$ (reference range, 0-500 ng/mL), an elevated C-reactive protein level of $6.16 \mathrm{mg} / \mathrm{dL}$ (reference range, $0-0.70 \mathrm{mg} / \mathrm{dL}$ ), and an elevated ferritin level of $3800 \mathrm{ng} / \mathrm{mL}$ (reference range, $20-250 \mathrm{ng} / \mathrm{mL}$ ). The result of a Novel Coronavirus (2019-nCoV) Real-Time polymerase chain reaction (RT-PCR) test via nasopharyngeal swab was positive.

\section{Diagnostic testing}

A chest radiograph revealed diffuse patchy opacities in the bilateral lung fields (Figure 1). The patient was admitted to the hospital for hypoxic respiratory failure caused by COVID-19 pneumonia and was treated with a 5-day course of dexamethasone, $10 \mathrm{mg}$, every 12 hours for

\section{AFFILIATIONS:}

'Department of Medicine, Division of Allergy/Immunology and Infectious Disease, Rutgers Robert Wood Johnson Medical School, New Brunswick, New Jersey

${ }^{2}$ Department of Medicine, Rutgers Robert Wood Johnson Medical School, New Brunswick, New Jersey

\section{CITATION:}

Solanki K, Kohm K, Bhatt PJ. Disseminated herpes zoster virus in a patient with COVID-19. Consultant. Published online April 8, 2021. doi:10.25270/con.2021.04.00003

Received October 23, 2020. Accepted January 7, 2021.

\section{DISCLOSURES:}

The authors report no relevant financial relationships.

\section{CORRESPONDENCE:}

Kinjal Solanki, MD, Rutgers Robert Wood Johnson Medical School, Clinical Academic Building 125, Paterson Street, New Brunswick, NJ 08901 (kinsolan@rwjms.rutgers.edu)

possible cytokine release syndrome. He also received hydroxychloroquine suspension, $400 \mathrm{mg}$, every 24 hours; enoxaparin sodium, $100 \mathrm{mg}$, every 12 hours; and 2 doses of tocilizumab. His interleukin- 6 level was $400 \mathrm{pg} / \mathrm{mL}$, which is more than 200 times the upper limit of normal.

Subsequently, he developed leukocytosis with bandemia, which was treated with intravenous vancomycin and piperacillin/tazobactam for suspected secondary bacterial infection. Blood cultures were negative, but a respiratory culture showed methicillin-susceptible Staphylococcus aureus, which was believed to be colonization. He completed a 7-day course of antibiotics for suspected superimposed bacterial pneumonia.

His hospital course was complicated by metabolic encephalopathy and acute kidney injury requiring renal replacement therapy, acute respiratory distress syndrome, and ventilator-dependent respiratory failure requiring tracheostomy and placement of a percutaneous endoscopic gastrostomy (PEG) tube. A computed tomography scan of the chest with contrast showed bilateral patchy ground glass opacities and small bilateral pleural effusions greater on the left (Figure 2).

The patient received antibiotics for septic shock of unknown etiology. He later developed fevers due to Clostridioides difficile infection, which was diagnosed by a positive stool toxin assay, and was treated with oral vancomycin, $125 \mathrm{mg}$, every 6 hours. His fevers resolved within 2 days of initiation of vancomycin.

His hospital course was further complicated by recurrent episodes of fever due to Pseudomonas aeruginosa bacteremia, which cleared quickly and was treated 


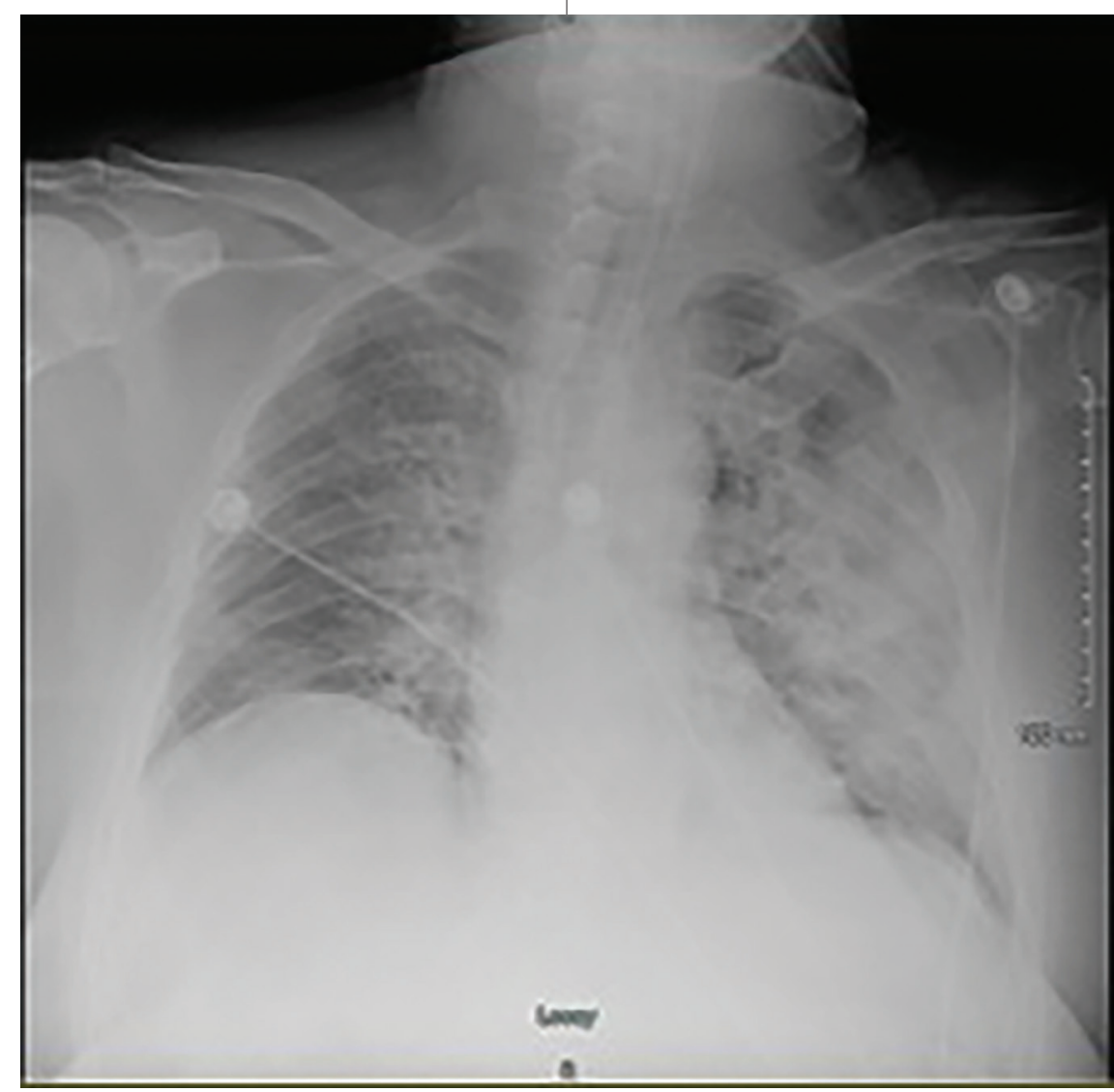

Figure 1. The anteroposterior view of the chest radiograph shows diffuse patchy opacities in the bilateral lung fields.

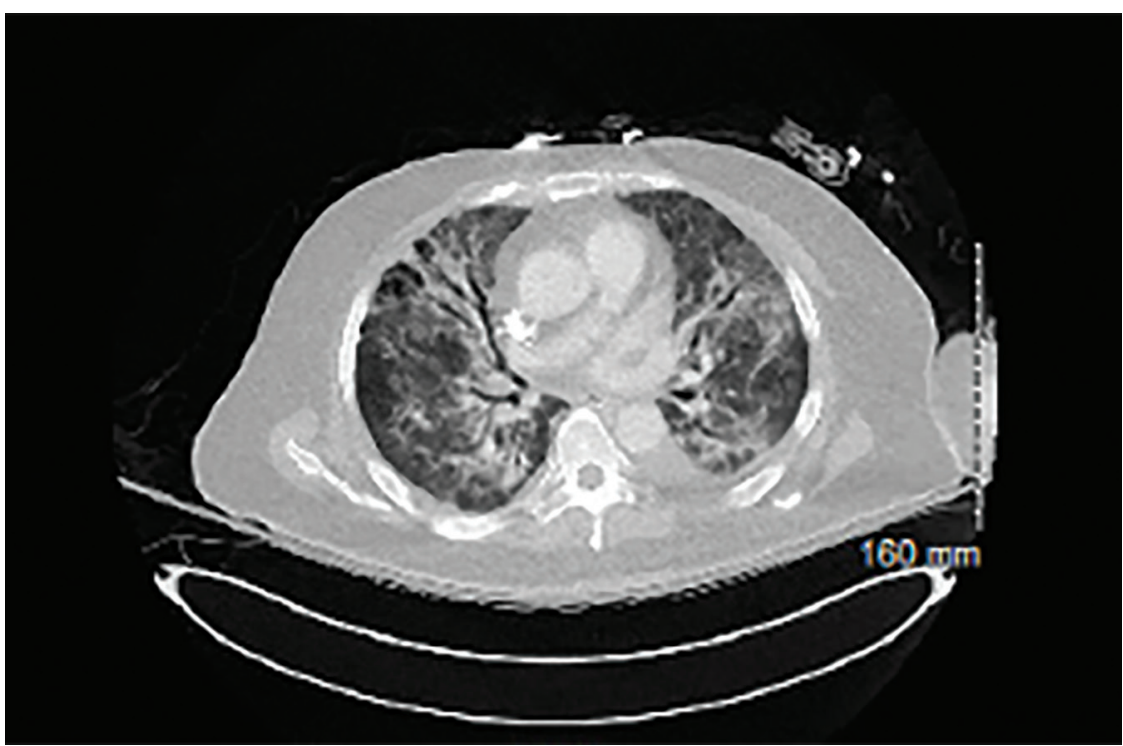

Figure 2. A computed tomography scan of the chest with contrast showed bilateral patchy ground glass opacities and small bilateral pleural effusions greater on the left. with 10 days of antibiotics ( 5 days of meropenem followed by 5 days of levofloxacin) with resolution of the fever.

A few days after the patient completed the antibiotic regimen, he developed another episode of fevers, and left-sided lung infiltrates were identified on a repeat chest radiography. $A$ tracheal aspirate culture revealed multidrug-resistant $P$ aeruginosa, which was treated with 7 days of ceftolozane/tazobactam for ventilator-associated pneumonia. $A$ T2Candida ${ }^{\circledR}$ panel PCR blood test was positive for Candida albicans, and the patient underwent proline removal and permcath exchange over guidewire, as his lines were a potential source of fungemia. He was treated with a 14-day course of micafungin, $100 \mathrm{mg}$, daily. The patient's fevers resolved within 3 days of initiation of the antifungal agents.

The patient remained afebrile for 5 days but subsequently developed a high-grade fever with a generalized rash (Figure 3). The rash appeared as fluid-filled blisters of varying stages all over his body, including on his face, with honey-colored crusting noted on the face (Figure 4).

At the time, the patient was diaphoretic and had tachycardia with diminished breath sounds in the anterior lung fields, but there were no noted changes in secretions. A chest radiograph showed worsening opacities in the right lung base, which were suspected to be microaspirations (Figure 5).

Tzanck preparation of skin scraping showed rare multinucleated giant cells. $A$ culture for the fluid from the blisters was positive for varicella-zoster virus (VZV); the results of a herpes simplex virus PCR test and bacterial cultures were negative. A VZV immunoglobulin G ( $\mathrm{g} G$ G) antibody test was positive, with an antibody index value of 2.91 (reference range, $<0.90$ ). The patient was placed on airborne and contact isolation and was empirically started on intravenous acyclovir, $2.5 \mathrm{mg} /$ kg, every 24 hours (renally dosed). Later, he was transitioned to a 2-week course of valacyclovir, $500 \mathrm{mg}$, via PEG tube every 


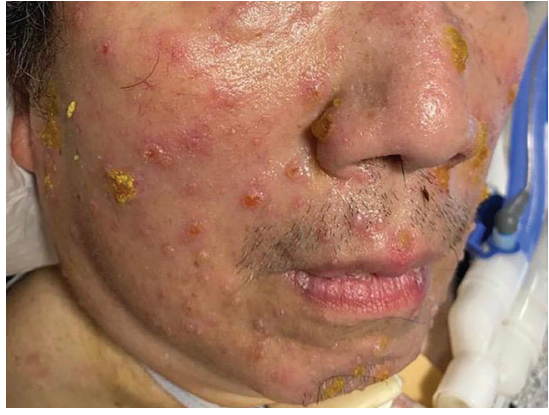

Figure 3. Honey-colored, crusted blisters in various stages were noted on the face.

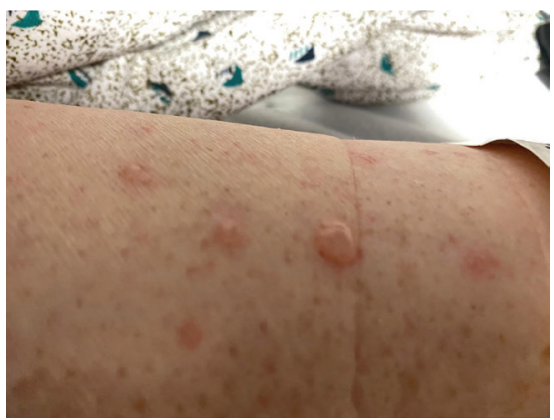

Figure 4. Fluid-filled blisters were noted on the right upper extremity.

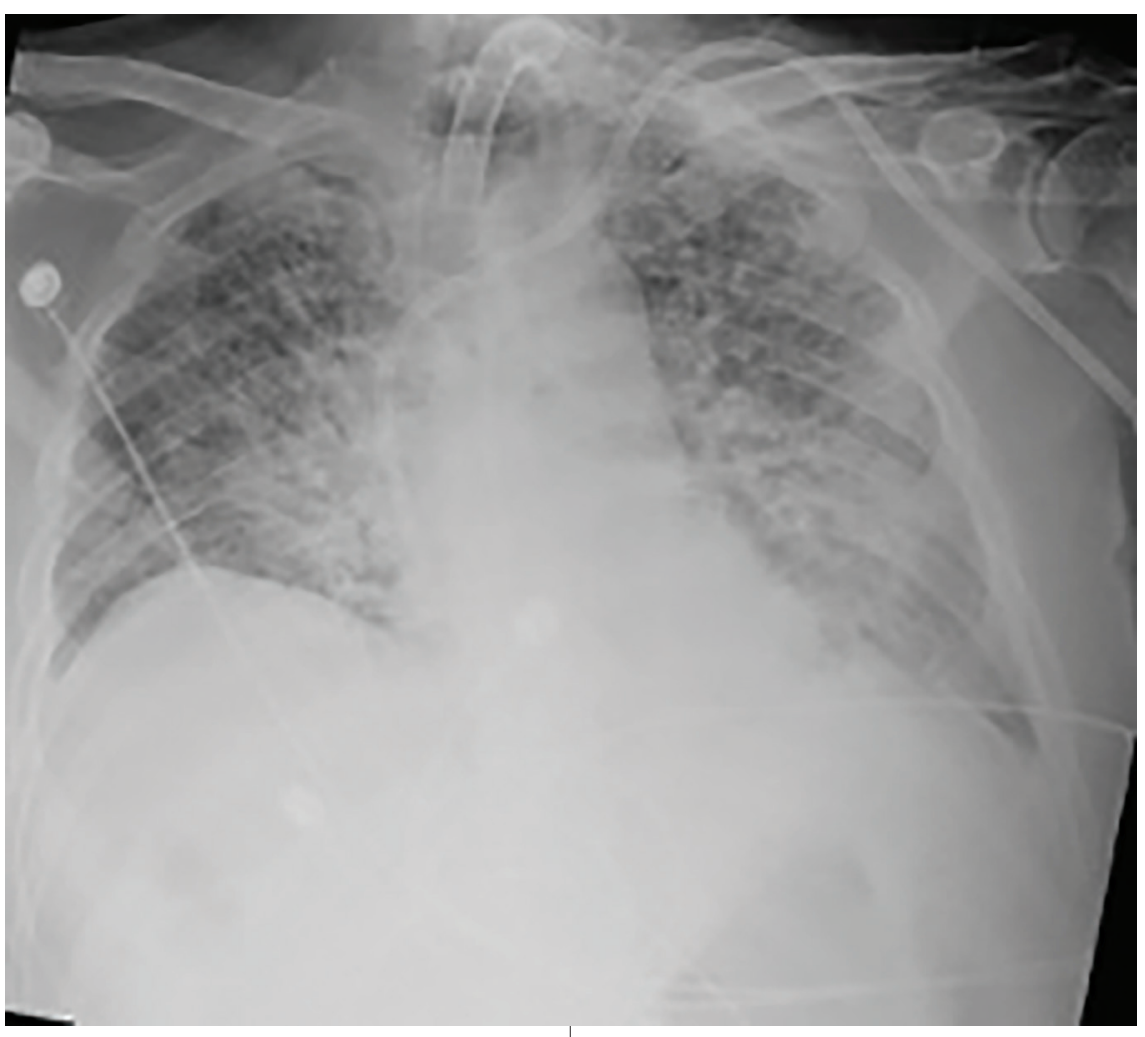

Figure 5. The anteroposterior view of the chest radiograph shows worsening opacities in the right lung base.
24 hours for disseminated herpes zoster infection. This treatment improved his skin lesions.

Severe acute respiratory disease virus-2 (SARS-CoV-2), or COVID-19, has infected more than 127 million people and has caused more than 2.7 million deaths worldwide as of March 2021. ' COVID-19 can present with a wide spectrum of manifestations. Skin manifestations associated with COVID-19 have been reported in the range of $0.2 \%$ (in Wuhan) to $20.4 \%$ (in northern Italy) of cases and include urticaria, varicella-like vesicular lesions, acral lesions ("COVID toes"), maculopapular and morbilliform rashes, and erythema multiforme..$^{2-5}$

Dermatological complications with the eruption of painful vesicular lesions, ulceration, and crusting in a single dermatomal pattern suggestive of herpes zoster infection has also been reported. ${ }^{6}$ Although several isolated cases of herpes

\section{Discussion}

zoster and COVID-19 coinfections have been reported, ${ }^{6,7}$ we present a unique case of disseminated herpes zoster infection that manifested during the convalescent phase of COVID-19 illness.

VZV, the cause of chickenpox, can remain dormant in the dorsal root ganglia of the spinal cord following a primary chickenpox infection and can reactivate later in life, causing herpes zoster infection. ${ }^{8}$ Reactivation typically manifests as localized disease with painful vesicular eruptions involving 1 or 2 adjacent dermatomes but, in the case of disseminated zoster, can also cause widespread disease involving 3 or more dermatomes. ${ }^{8}$

Risk of VZV reactivation increases with age, with an incidence of 1 per 100 population in the United States annually in those aged 60 years or older, and is thought to be associated with immunosenescence. Immunocompetent individuals are typically afflicted with localized disease along 1 or 2 contiguous dermatomes. ${ }^{8}$ Disseminated disease primarily manifests among patients who are immunocompromised, including those with malignancy on chemotherapy, HIV, bone marrow and solid organ transplant, and those on chronic immunosuppressive medications. ${ }^{8}$

Since the first time SARS-CoV-2 virus was isolated in Wuhan, China, in January 2020, much has been reported regarding epidemiological risk factors and laboratory findings of these patients and their association with mortality. ${ }^{9,10}$ Findings such as lymphopenia and low percentage of CD4+ T cells, CD8+ T cells, B cells, and natural killer cells have been associated with increased mortality in patients with COVID-19. ${ }^{9,10}$ Functional impairment of CD4+ $T$ cells has also been reported, in addition to exhaustion of CD8 $+T$ cells from excessive activation, both of which can predispose patients to reactivation of VZV virus in a severe form, ${ }^{11,12}$ as observed in our patient who had no other underlying immunocompromised risk factors.

Since there have been reports of 
herpes zoster coinfection in patients with COVID-19, 7,11 shingles vaccination is encouraged per the Centers for Disease Control and Prevention guidelines for all adults aged 50 years or older who have had a history of chickenpox or shingles. ${ }^{8}$

\section{Patient outcome}

His skin lesions fully crusted 7 days after the initiation of antiviral agents. His fever resolved, and he was discharged to a long-term acute care facility, where he eventually completed his 2-week antiviral treatment course.

\section{REFERENCES}

1. Dong E, Du H, Gardner L. An interactive web-based dashboard to track COVID-19 in real time. Lancet Infect Dis. 2020;20(5):533534. https://doi.org/10.1016/S14733099(20)30120-1

2. Guan WJ, Ni ZY, Hu Y, et al; China Medical Treatment Expert Group for COVID-19. Clinical characteristics of coronavirus disease 2019 in China. N Engl J Med. 2020;382(18):1708-1720. https://doi. org/10.1056/NEJMoa2002032

3. Recalcati S. Cutaneous manifestations in
COVID-19: a first perspective. J Eur Acad Dermatol Venereol. 2020;34(5):e212-e213. https://doi.org/10.1111/jdv.16387

4. Wollina U, Karadağ AS, Rowland-Payne C, Chiriac A, Lotti T. Cutaneous signs in COVID-19 patients: a review. Derm Ther. 2020;33(5):e13549. https://doi.org/10.1111/ dth.13549

5. Hernandez C, Bruckner AL. Focus on "COVID toes." JAMA Dermatol. 2020;156(9):1003. https://doi.org/10.1001/ jamadermatol.2020.2062

6. Elsaie ML, Youssef EA, Nada HA. Herpes zoster might be an indicator for latent COVID 19 infection. Dermatol Ther. 2020;33(4):e13666. https://doi.org/10.1111/ dth.13666

7. Elsaie ML, Nada HA. Herpes zoster (shingles) complicating the course of COVID19 infection. J Dermatolog Treat. Published online October 12, 2020. https://doi.org/10.1080/09546634.2020.17 82823

8. Shingles (herpes zoster). Centers for Disease Control and Prevention. Reviewed October 5, 2020. Accessed October 21, 2020. https://www.cdc.gov/shingles/ hcp/clinical-overview.html
9. Zhou F, Yu T, Du R, et al. Clinical course and risk factors for mortality of adult inpatients with COVID-19 in Wuhan, China: a retrospective cohort study. Lancet. 2020;395(10229):1054-1062. https://doi. org/10.1016/S0140-6736(20)30566-3

10. Huang $C$, Wang $Y, L i X$, et al. Clinical features of patients infected with 2019 novel coronavirus in Wuhan, China. Lancet. 2020;395(10223):497-506. https://doi. org/10.1016/S0140-6736(20)30183-5

11. Saati A, Al-Husayni F, Malibari AA, Bogari AA, Alharbi M. Herpes zoster co-infection in an immunocompetent patient with COVID-19. Cureus. 2020;12(7):e8998. https://doi.org/10.7759/cureus.8998

12. Zheng HY, Zhang M, Yang CX, et al. Elevated exhaustion levels and reduced functional diversity of $\mathrm{T}$ cells in peripheral blood may predict severe progression in COVID-19 patients. Cell Mol Immunol. 2020;17(5):541-543. https://doi. org/10.1038/s41423-020-0401-3 